\title{
Aportes del trabajo colaborativo interinstitucional en procesos de extensión para la transformación social: caso Ugrosanta
}

\section{Contributions of Inter-Institutional Collaborative Work in Extension Processes for Social Transformation: The Ugrosanta Case}

Marta Vargas Venegas Universidad Nacional Heredia, Costa Rica marta.vargas.venegas@una.ac.cr https://orcid.org/0000-0003-4496-9908

Alejandra Ávila Artavia

Universidad Nacional Heredia, Costa Rica

alejandra.avila.artavia@una.cr https://orcid.org/0000-0002-0843-1433

Priscilla Mena García Universidad Nacional Heredia, Costa Rica priscila.mena.garcia@una.cr https://orcid.org/0000-0001-7632-0594

Recibido:06/10/2020 - Aceptado: 27/01/2021

Received: 06/10/2020 • Accepted: 27/01/2021

Resumen. Este trabajo se efectúa en el marco del quehacer de la Escuela de Planificación y Promoción Social, de la Facultad de Ciencias Sociales de la Universidad Nacional (UNA), Costa Rica. Dicha unidad académica surge en el año 1974 y desde sus inicios se ha caracterizado por integrar prácticas de extensión en los procesos de docencia, propiciando la participación activa del estudiantado, del equipo académico, de las comunidades y de las instituciones, a través de un trabajo colaborativo que busca la interacción de diferentes actores sociales, en pro de la transformación social.

Se presentan resultados de un trabajo colaborativo de tres años desarrollado en la comunidad de Santa Cruz de Turrialba, Costa Rica, en coordinación con el Sistema 
Revista Universidad en Diálogo • Vol. 11, N. 2 2, Julio-Diciembre, 2021 • 69-94

ISSN 2215-2849 • EISSN: 2215-4752

URL: http://www.revistas.una.ac.cr/index.php/dialogo/index Correo electrónico: universidadendialogo@una.cr DOI: http://doi.org/10.15359/udre.11-2.4

Nacional de Áreas de Conservación (Sinac), la organización Unión de Agrupaciones de Santa Cruz de Turrialba (Ugrosanta), quince personas estudiantes y dos profesoras de la Escuela de Planificación y Promoción Social (EPPS). Además, el proyecto contó con la participación del Ministerio de Cultura y Juventud de Costa Rica.

Primeramente, se señalan conceptos que son claves para este análisis, como el trabajo colaborativo, la extensión universitaria y el desarrollo rural con enfoque territorial como base de la transformación social. Después, se contextualiza el caso de estudio, así como la metodología utilizada para la elaboración del presente artículo. Por último, se exponen los resultados de las entrevistas realizadas y las reflexiones académicas que genera el desarrollo de la relación universidad-sociedad mediante el trabajo colaborativo.

Palabras clave: trabajo colaborativo, extensión universitaria, transformación social.

Abstract. This work is carried out by the School of Planning and Social Promotion, which is part of the Faculty of Social Sciences of the National University of Costa Rica (UNA). This academic unit was created in 1974; it has been characterized by integrating extension practices in teaching processes and promoting the active participation in students, academic teams, communities, and institutions through collaborative work that seeks the interaction between different social actors in favor of social transformation.

This article presents the results of a 3-year collaborative work, developed in the community of Santa Cruz de Turrialba, Costa Rica, in coordination with the National System of Conservation Areas (SINAC), the organization Unión de Agrupaciones de Santa Cruz de Turrialba (UGROSANTA), fifteen students, and two teachers from the School of Planning and Social Promotion (EPPS) of the National University. In addition, the project had the participation of the Ministry of Culture and Youth of Costa Rica.

First, key concepts for this analysis are highlighted, such as collaborative work, university extension, and rural development with a territorial approach as a basis for social transformation. Subsequently, the case study and the methodology used to write this article are contextualized. Finally, the results of the interviews conducted and the academic reflections generated by the development of the university-society relationship through collaborative work are presented.

Keywords: collaborative work, university extension, social transformation.

\section{Introducción}

Las instituciones públicas (del Estado) buscan generar acciones que se sustenten en el trabajo directo con las personas que son beneficiarias de sus servicios. Esta práctica fue muy común dentro del Estado benefactor en Costa Rica (1948-1980) y permanece amparada en uno de los poderes y derechos reconocidos a la sociedad, que se reconocen hoy en la Constitución Política como el derecho a la participación ciudadana. Algunas instituciones realizan 
trabajo de campo en busca de cumplir con esa vinculación esperada entre sus acciones y el contexto al que son dirigidas.

La universidad pública ha sido pensada, desde el imaginario colectivo, como benefactora de la sociedad, pues representa la oportunidad para que las personas estudien y con ello logren incorporarse al mercado laboral en mejores condiciones. Además, la universidad asume otros compromisos, como generar conocimiento a través de la investigación y de la extensión.

La extensión promueve una vinculación directa y una relación dialógica de estudiantes y docentes con la sociedad, acciones que no solo aportan nuevos conocimientos, sino que además fortalecen procesos de aprendizaje construidos desde la práctica y el compromiso social.

Es común que instituciones y universidades coincidan en sus trabajos de vinculación y relación comunitaria dentro de un territorio, y en ello es posible que se presenten dos situaciones: 1. Trabajan el mismo tema o similares, pero con enfoques e intereses particulares, con tiempos y recursos diferentes. 2 . Trabajan temáticas diferentes.

En el caso que sirve de estudio para este artículo, la vinculación entre la UNA y el Sinac se dio antes de realizar el trabajo de campo en Santa Cruz de Turrialba. A pesar de que ambas instituciones trabajaron el desarrollo territorial desde las comunidades, sus enfoques iniciales fueron diferentes (el del Sinac de transferencia de conocimiento y el de la UNA de construcción colectiva). Sin embargo, se fueron ajustando a partir de la dinámica del diálogo y el establecimiento del vínculo con Ugrosanta, como organización del territorio.

Las particularidades de este proceso al que se denomina trabajo colaborativo, así como las variables que confluyeron para que se lograra, se presentan en este artículo, y con ello se invita a una reflexión que motive a las instituciones a consolidar sus procesos de campo de forma coordinada con diversos actores, que tengan presencia o estén interesados en brindar condiciones que generen bienestar para las personas del territorio.

\section{Referente teórico}

\section{Trabajo colaborativo interinstitucional}

Existen diversidad de acepciones que se asocian con el trabajo colaborativo, las cuales representan una ampliación y/o profundización del trabajo en equipo y se desarrollan principalmente dentro de espacios de enseñanza y aprendizaje 
Revista Universidad en Diálogo • Vol. 11, N. 2 2, Julio-Diciembre, 2021 • 69-94

ISSN 2215-2849 • EISSN: 2215-4752

URL: http://www.revistas.una.ac.cr/index.php/dialogo/index CorReo ElECTRÓNICO: universidadendialogo@una.cr DOI: http://doi.org/10.15359/udre.11-2.4

donde se reconoce como la oportunidad de incrementar los conocimientos y las capacidades de las personas involucradas (estudiantes y docentes).

El economista y catedrático Yochai Benkler (2006) se refiere al concepto desde una posición de transformación de la economía y los intercambios entre productores y consumidores, donde los segundos pasan de un papel pasivo (receptor) a uno activo (co-creador) en la definición del tipo de servicios y productos que requieren.

Su profundización se realiza en referencia a las herramientas digitales (internet, Wikipedia, Google Drive, entre otros). Benkler define en su libro La riqueza de las redes el trabajo colaborativo o procomún como "el sistema de producción, distribución y consumo de bienes de información que se caracteriza por acciones individuales descentralizadas, ejecutadas a través de medios ampliamente distribuidos y ajenos al mercado y a sus estrategias" (2016, p. 345).

Dentro de esta definición se resaltan elementos vinculados a procesos de autodefinición, autogestión y autonomía de las partes que interactúan dentro de los espacios de trabajo colaborativo, para la consecución de un fin común mediante la cooperación individual, entendida como el aporte de experiencias, conocimientos, percepciones y perspectivas para el enriquecimiento de todos y todas.

Se entiende que el trabajo colaborativo encuentra vida en espacios o procesos compartidos por personas u organizaciones heterogéneas, pero con ideales y objetivos comunes. Seresalta en esta interacción la importancia de la comunicación, la coordinación, la negociación y la concertación como factores de éxito.

Existen cinco elementos que deben tenerse en cuenta para el trabajo colaborativo:

1. Interdependencia positiva: Los y las integrantes de un grupo persiguen un objetivo común y comparten recursos e información.

2. Promoción de la interacción: Las personas integrantes de un grupo se ayudan unas a otras para trabajar eficiente y efectivamente mediante la contribución individual de cada participante.

3. Responsabilidad individual: Cada una de las personas del grupo es responsable por su aporte individual y por la manera en la que ese aporte contribuye al aprendizaje de todos y todas.

4. Habilidades y destrezas de trabajos grupales: Cada persona debe comunicarse, apoyar a las demás personas y resolver conflictos con otras personas participantes, constructivamente. 
5. Interacción positiva: Cada persona debe mantener una buena relación de cooperación con las demás personas y estar dispuesta a dar y recibir comentarios y críticas constructivas sobre sus contribuciones. (Barragán de Anda, De Aguinaga y Ávila, 2010)

Hacer referencia a este concepto implica tener conciencia sobre el conjunto de variables que intervienen de manera simultánea en la configuración y los resultados del trabajo colaborativo. Incorpora elementos que van desde actitudes y capacidades individuales hasta condiciones institucionales y organizacionales, como incentivos y prácticas relacionadas con el diálogo constante e intercambios de experiencias y conocimientos que facilitan u obstaculizan su desarrollo.

Para el caso del trabajo colaborativo institucional, se parte de los elementos antes planteados y se suma la disposición de las personas representantes de las instituciones que participan en los procesos, como elemento fundamental desde etapas iniciales, ya que además propicia la permanencia de procesos de construcción conjunta a lo largo del tiempo.

El aporte de este concepto al presente artículo se asocia con el enriquecimiento de los procesos de extensión tras la articulación de diferentes actores, ya que se generan interaprendizajes y se propicia la construcción de un marco teórico e interpretativo común y con sentido para todos y todas. Ello se logra a partir de la relación que se configura entre la organización comunal, las instituciones públicas, las personas docentes y la población estudiantil.

Cada persona aporta experiencias, conocimientos, visiones y sentimientos que se convierten en insumos valiosos dentro del proceso, que además nunca termina, ya que tiene un comportamiento cíclico, pues las experiencias, los conocimientos, las visiones y los sentimientos evolucionan constantemente debido a la relación y comunicación constante, logrando que el proceso no deje de enriquecerse.

Se entiende dentro de este proceso a la organización comunal como un conjunto de personas que se unen alrededor de intereses y objetivos compartidos, y que se encuentran dentro de un espacio geográfico definido, el cual es denominado como comunidad. Según Torres (2013), se entiende por comunidad a una "categoría analítica y política para comprender y encauzar procesos y proyectos comunitarios en un horizonte emancipatorio" (p. 9).

Aunque se relaciona con lo común y con la unión, lo cierto es que en la comunidad existe heterogeneidad, diferencia, pluralidad y por ello nacen las organizaciones que, dentro de ese contexto diverso, coinciden en valores, ideales y metas, que motivan el trabajo de forma conjunta. 
Revista Universidad en Diálogo • Vol. 11, N. 2 2, Julio-Diciembre, 2021 • 69-94

ISSN 2215-2849 • EISSN: 2215-4752

URL: http://www.revistas.una.ac.cr/index.php/dialogo/index CorReo ElECTRÓNICO: universidadendialogo@una.cr DOI: http://doi.org/10.15359/udre.11-2.4

En Costa Rica existe una clasificación de instituciones públicas según su naturalezajurídica, entre las cuales se encuentran: ministerios, órganos adscritos, instituciones autónomas, semi-autónomas, empresas públicas, entes públicos no estatales, municipalidades, tribunales electorales y poderes de la República. Para definir la clasificación se toma como referencia a la Constitución Política de la República de Costa Rica (CPRCR), la Ley Orgánica o constitutiva, el Reglamento Orgánico y el criterio de la Procuraduría General de la República (Mideplan, 2010).

Se entiende por consiguiente a la institución pública como aquella organización que nace como respuesta a una necesidad identificada por el Estado y la cual opera mediante fondos públicos y no tiene fines lucrativos; además posee como fin el bienestar de las personas en diferentes áreas socioeconómicas.

\section{Extensión universitaria: un pilar de la Universidad Nacional}

Desde sus inicios, la Universidad Nacional se propuso considerar decisiones sobre la educación formativa, la investigación y la extensión universitaria, referida en aquel momento a los servicios que debía presentar la Universidad a la comunidad. Para ello el Padre Núñez estableció en el documento denominado Hacia una Universidad Necesaria (1974) lo siguiente:

Téngase presente que tales servicios deben superar decididamente la "extensión universitaria" tradicional, concebida y practicada como una entrega ocasional de migajas del saber a públicos muy restringidos, o como actitud filantrópica del vulgarización, atomización y degradación de la cultura para consumo popular. Esa concepción elitista debe ser radicalmente sustituida por la actitud que busca y confia hacer que el pueblo actúe como sujeto de su propia historia y de su propia libertad. (p. 33)

Esta cita textual deja claro que era necesario el establecimiento de objetivos que permitieran la participación activa de la población en lo referente a la expresión y la auto-organización de los diferentes actores sociales en pro del desarrollo del país.

Es en este contexto que en el año 1974 surge la Escuela de Planificación y Promoción Social (EPPS), en la cual se desarrolla el primer proyecto de extensión universitaria de la UNA, el mismo estaba dirigido a colaborar en el desarrollo de la Federación de Empresas Comunitarias, que buscaba ser una alternativa de organización para las personas asalariadas temporales del agro (Sobrado, 1976). 
Es así como desde su génesis la EPPS ha incorporado el pilar de la extensión no solo en su quehacer desde los programas y proyectos, sino en el Plan de Estudios, considerando la participación y el diálogo de saberes como ejes claves en la contribución del desarrollo de las comunidades y de la misma Universidad.

La base filosófica de la UNA se sustenta en la dinámica del cambio social, con el fin de otorgar a la sociedad medios a través de los cuales se pueda repensar crítica y científicamente su experiencia acumulada. Su quehacer ha estado conformado por cuatro pilares: la docencia, la extensión, la investigación y la producción.

En el caso de la extensión, esta en sus inicios se consideró como aquella área sustantiva "...que permite conocer la realidad, adquirir una visión totalizadora enriqueciendo y direccionando la actividad académica en su conjunto, en busca de la liberación y transformación social" (Sánchez, 1989, p. 4).

Actualmente, desde la Universidad Nacional, se conceptualiza la extensión universitaria como

Una tarea por medio de la cual, la universidad se interrelaciona con la comunidad nacional de manera crítica y creadora. Por medio de ésta proyecta a la sociedad los productos del quehacer académico, lo que permite que se redimensione y enriquezcan al percibir las necesidades de la sociedad, materializándose el vínculo universidad-sociedad. (Universidad Nacional, 2018)

En este planteamiento se evidencia la interrelación de la universidad con la comunidad, recordando que la concepción de la universidad necesaria establecía la importancia de interactuar con la realidad social, promoviendo procesos participativos de aprendizaje que permitieran desde las comunidades proponer acciones innovadoras y socialmente satisfactorias, en pro de la transformación social de los diferentes territorios del país.

\section{Desarrollo rural con enfoque territorial: hacia la transformación social}

Para abordar adecuadamente el desarrollo rural con enfoque territorial es preciso definir desarrollo y territorio.

El concepto de desarrollo se ha transformado a lo largo de la historia. Una de sus primeras aproximaciones, en la década de los cuarenta, se concentró, según Dubois (2014), en descifrar las causas y los medios para alcanzar el crecimiento económico, en tanto que nuevas posturas se inclinan por atender los objetivos y fines del desarrollo. 
Revista Universidad en Diálogo • Vol. 11, N. 2 2, Julio-Diciembre, 2021 • 69-94

ISSN 2215-2849 • EISSN: 2215-4752

URL: http://www.revistas.una.ac.cr/index.php/dialogo/index CORREO ELECTRÓNICO: universidadendialogo@una.cr DOI: http://doi.org/10.15359/udre.11-2.4

En 1990 Amartya Sen realiza importantes aportes en el primer Informe sobre Desarrollo Humano del Programa de las Naciones Unidas, pues agrega la perspectiva social, más allá de la económica, para medir el desarrollo. Señala el informe que "el fin del desarrollo debe ser el bienestar humano. La manera de relacionar los medios con el fin último debe convertirse nuevamente en el aspecto central del análisis y de la planeación para el desarrollo" (PNUD, 1990, p. 33). De esta manera, define que "el desarrollo humano es un proceso mediante el cual se amplían las oportunidades de los individuos..." (p. 33).

Sen, por su parte, asocia el nivel de vida con los conceptos de capacidades, realizaciones y bienes y servicios. Las realizaciones son vistas como las condiciones de vida que pueden ser alcanzadas o no, en tanto las capacidades se muestran como la habilidad para alcanzar dichas condiciones. Por otro lado, el poseer bienes y servicios no es considerado un criterio para definir el nivel de vida (citado por Bolívar, 2009). A partir de ese momento la participación de las instituciones en el desarrollo se vuelve importante.

Paralelamente, con la publicación del Informe de Brundtland de la Comisión Mundial de Medio Ambiente de Naciones Unidas en 1987 se incorpora la definición del concepto de desarrollo sustentable, dando importancia al medio ambiente, de forma tal que se satisfagan las necesidades sin comprometer la capacidad de las generaciones futuras. Esto queda luego ratificado en la Declaración de Río o Agenda 21, a partir de la Conferencia de las Naciones Unidas sobre el Medio Ambiente y el Desarrollo en Río de Janeiro celebrada en 1992, que señala el derecho que tiene el ser humano de vivir saludablemente en armonía con la naturaleza.

Posteriormente, se visualiza la necesidad de generar acciones concretas y se formulan los Objetivos de Desarrollo del Milenio (ODM) con estrategias al 2015 orientadas a temas de salud, pobreza, educación, equidad y ambiente (Picazzo et al., 2011). Finalmente, la nueva agenda, llamada ahora de desarrollo sostenible, define los objetivos globales (ODS) 2015-2030 que se amplían en temas como agua, energías, ecosistemas, clima, industria, etc. (ONU, s. f.).

El concepto de desarrollo ha ampliado su alcance tomando un abordaje multidimensional, particularmente porque se da mayor importancia al fin, asociado con el bienestar humano y la sostenibilidad, y por ende con todos aquellos aspectos que lo afectan.

Sepúlveda y Zúñiga (2008) los identifican como factores de orden sociocultural, económico, ambiental y político-institucional. Este enfoque basado en el fin 
URL: http://www.revistas.una.ac.cr/index.php/dialogo/index

CORREO ELECTRÓNICO: universidadendialogo@una.cr

DOI: http://doi.org/10.15359/udre.11-2.4

y no en el objeto mismo evidentemente conlleva a la necesidad de trazar estrategias hacia una transformación que busca el mayor beneficio para la sociedad (las personas).

Bajo este mismo enfoque, el territorio es visto como una unidad de planificación y gestión que articula en distintos espacios: lo local, regional, nacional e internacional. Esta visión multiescalar toma en cuenta factores endógenos y exógenos que influyen o condicionan el desarrollo de los territorios rurales. Lo anterior busca cumplir con dos objetivos primordiales: la cohesión social y la territorial. Esta cohesión se basa en "...la construcción de la sociedad sustentada en la equidad y sentido de pertenencia; que integre los territorios a una visión país. Para lograrlo, se recurre a la coordinación y articulación de instituciones públicas y el ámbito privado, mediado por actores organizados en los territorios" (Ávila y Aguilar, 2020).

Ávila (2013) reflexiona acerca de la importancia de que el desarrollo rural incorpore las propuestas de todos los sectores (multisectorialidad) en la formulación de políticas públicas, estrategias y planes, a fin de lograr una sostenibilidad integral apoyada en principios de gobernanza y participación ciudadana. La autora cobija el enfoque de la nueva ruralidad, el cual surge en los años noventa y aspira a un espacio donde se puedan diversificar las actividades económicas y oportunidades de empleo, una visión que supera lo estrictamente agropecuario y promueve un mayor dinamismo territorial en línea con el beneficio colectivo.

De acuerdo con el Plan Nacional de Desarrollo Rural Territorial, Costa Rica, 2017-2022 (Instituto de Desarrollo Rural, 2017), además de los principios de multidimensionalidad, multiescalaridad y multisectorialidad, en Costa Rica se procura cumplir el criterio de diferencialidad, que busca potenciar y aprovechar las particularidades de cada territorio, bajo un marco de acciones que se puedan ajustar a diferentes realidades.

Si se piensa en temas de desarrollo comunitario y el enfoque territorial es importante ubicarnos, primeramente, en la definición de comunidad. Para Zárate (citado por Carvajal, 2011), muchas de las posturas en torno al término comunidad coinciden en algunos elementos: "Es un conjunto de personas. Comparten algún interés, rasgo o elemento. Se ubican en una determinada área geográfica. Tienen conciencia de pertenencia. Se da una interacción intensa entre los miembros. Constituye una unidad social" (p. 23). Asimismo, Montero (citado por Carvajal, 2011) agrega el aspecto dinámico como elemento intrínseco de la comunidad, es decir, la comunidad está en constante transformación. 
Revista Universidad en Diálogo • Vol. 11, N. 2 2, Julio-Diciembre, 2021 • 69-94

ISSN 2215-2849 • EISSN: 2215-4752

URL: http://www.revistas.una.ac.cr/index.php/dialogo/index CORREO ELECTRÓNICO: universidadendialogo@una.cr DOI: http://doi.org/10.15359/udre.11-2.4

Lo anterior coincide con lo señalado por Camacho (2013), quien anota que las características básicas que debe tener una comunidad son: una dimensión territorial o localidad, un colectivo de personas que allí conviven y un espacio sentido como propio, lo cual genera identidad y participación social.

Respecto a la dimensión territorial o localidad, Schejtman y Berdegué (2004) precisan que:

El territorio no es un espacio físico "objetivamente existente", sino una construcción social, es decir, un conjunto de relaciones sociales que dan origen y a la vez expresan una identidad y un sentido de propósito compartido por múltiples agentes públicos y privados. (p. 4)

Mançano (2009) plantea que los tipos de territorios se definen, más allá de un simple espacio de gobierno, en función de las relaciones sociales y por tanto de un espacio relacional. Por otro parte, señala este autor que en el principio de multidimensionalidad se ve al territorio como una totalidad que integra espacio geográfico y relaciones sociales.

Asimismo, la multiescalaridad abre la posibilidad de pensar los territorios en espacios de gobernanza a diferentes niveles, desde lo nacional hasta lo local, y ello genera, además, grados de conflictualidad que nacen de la heterogeneidad y diferencialidad de los territorios. Particularmente, Mançano enfatiza en una visión integral de la tipología del territorio como “...espacio de gobernanza, como propiedades y como espacio relacional" (p. 17).

Una de las preocupaciones, y por tanto retos, que la Universidad Nacional identificó en la formulación de su Plan de Mediano Plazo 2017-2021 fue la visualización fragmentada de la acción territorial. De allí la necesidad de vincular dentro de sus metas el abordaje del ámbito de acción territorial desde una perspectiva articulada e integral, lo cual es coincidente con la visión de totalidad que plantean diversas personas autoras.

\section{Contextualización del caso de estudio}

Este caso se asocia con la experiencia generada a partir del proyecto desarrollado en el marco del curso Práctica Organizativa I y II, que pertenece a la malla curricular de la carrera Planificación Económica y Social. Este es matriculado por estudiantes en el tercer año de formación universitaria y se desarrolla en un periodo de nueve meses. 
Aunque las personas estudiantes permanecen en las comunidades por un lapso establecido, el proceso puede extenderse con matrículas posteriores, incorporando al estudiantado. La decisión depende, por un lado, de la pertinencia académica y social de dar continuidad, así como del interés por parte de la comunidad u organización involucrada.

El programa del curso define lo siguiente:

La Práctica Organizativa es un espacio que contribuye a formar profesionales con la capacidad de "desarrollar procesos institucionales, organizativos y sociales acordes a las tendencias más relevantes de este momento, a saber: la participación comprometida de la sociedad civil, animación de procesos de descentralización y desconcentración y procesos de autogestión local y regional con involucramiento de los sujetos del desarrollo". (Informe evaluativo académico, 1998, p. 3).

Las prácticas comunitarias son reconocidas en diversos espacios y por parte de gran cantidad de actores locales e institucionales. En diversas ocasiones, tal como ocurrió en el caso de estudio, se generan alianzas formales e informales con instituciones o de base comunitaria.

El proyecto tuvo como punto de partida la solicitud realizada por el Sistema Nacional de Áreas de Conservación (Sinac), del Área de Conservación Cordillera Volcánica Central (ACCVC), a la EPPS para asignar estudiantes en proyectos comunitarios ubicados en el cantón de Turrialba, provincia de Cartago, en la Región Central de Costa Rica.

A principios del año 2016 se llevaron a cabo reuniones con el fin de conocer el interés del Sinac y el proyecto con el que eventualmente se establecería el compromiso y la participación estudiantil.

Dentro de los elementos de negociación inicial en este tipo de alianzas, se encuentran: el apoyo a las personas estudiantes (con hospedaje, alimentación, acceso a información, apertura para el desarrollo de talleres y otras actividades), así como el interés y compromiso de la contraparte institucional $\mathrm{y}$ de la comunidad u organización con la que se desea trabajar.

La intención inicial del Sinac consistía en atender dos organizaciones comunales con las que previamente había trabajado el Programa de las Naciones Unidas para el Desarrollo (PNUD) como ente financiador de iniciativas productivas y donde el Sinac era quien identificaba las iniciativas productivas que recibirían el beneficio económico. 
Revista Universidad en Diálogo • Vol. 11, N. 2 2, Julio-Diciembre, 2021 • 69-94

ISSN 2215-2849 • EISSN: 2215-4752

URL: http://www.revistas.una.ac.cr/index.php/dialogo/index CorReo ElECTRÓNICO: universidadendialogo@una.cr DOI: http://doi.org/10.15359/udre.11-2.4

Las dos iniciativas financiadas recibieron el dinero, compraron materiales y algunos activos; sin embargo, una vez agotados el dinero y los insumos, no lograron emprender o continuar con la actividad. Estas iniciativas se convirtieron en la base del proyecto de fortalecimiento organizacional que se trabajaría desde las prácticas estudiantiles.

En un segundo momento se realizó la gira para conocer estas organizaciones del cantón de Turrialba:

1. Una de ellas estaba conformada por mujeres que hacían manualidades con material reciclado y se ubicaba en la Pastora de Santa Cruz de Turrialba.

2. La otra estaba constituida por personas que trabajaban como guías turísticas de la zona, que provenían principalmente de Guayabo y de Santa Cruz centro. Ambas comunidades se dedican principalmente a la ganadería de leche y a la agricultura. Guayabo recibe, además, visitación turística por ubicarse ahí el Monumento Nacional Guayabo.

Se llevó a cabo un conversatorio con cada organización, el cual permitió a sus integrantes y a las personas docentes conocerse y conocer, además, la percepción y el interés en el proyecto de fortalecimiento organizacional, así como la contribución y el papel de la UNA y el Sinac en el proceso. Se identificó un interés común en el trabajo que se podría realizar de manera conjunta.

Iniciado el ciclo lectivo del año 2016, se asignaron estudiantes al proyecto y se realizaron reuniones con el funcionario encargado del Sinac, en las que se compartió con las personas estudiantes información sobre la idea de proyecto y lo que se esperaba que ellas impulsaran.

A ello siguieron giras de reconocimiento de la zona y, por último, sesiones conjuntas entre las organizaciones, el Sinac, estudiantes y personal académico. Paralelamente, el estudiantado elaboró una contextualización de la zona en las dimensiones económica, social, ambiental y político-institucional, lo que constituyó un diagnóstico participativo.

\section{Elaboración y análisis de los resultados del diagnóstico}

Este proceso se basó en la elaboración de un diagnóstico comunal con enfoque participativo, que fue ejecutado por estudiantes de planificación, con apoyo y asesoría de las académicas tutoras y retroalimentación del Sinac; 
URL: http://www.revistas.una.ac.cr/index.php/dialogo/index

CORREO ELECTRÓNICO: universidadendialogo@una.cr

DOI: http://doi.org/10.15359/udre.11-2.4

posteriormente, los resultados parciales fueron presentados y validados con una agrupación comunal de la zona.

Entendiendo el diagnóstico como un proceso de investigación aplicada que ofrece información para la solución de problemas o la transformación de una situación identificada.

Entre los pasos realizados de forma conjunta entre los y las estudiantes, el Sinac y el cuerpo docente se encuentran:

A. La delimitación del territorio sobre el cual se realizaría la investigación diagnóstica: Santa Cruz de Turrialba.

B. Se establecieron las dimensiones de estudio.

C. Se elaboraron y aplicaron entrevistas a instituciones, organizaciones y personas de la comunidad que en ese momento eran reconocidas por su trabajo y actividad en beneficio de la comunidad.

D. Se realizaron sesiones de trabajo del equipo para el análisis de la información recolectada mediante revisión documental, aplicación de entrevistas y fichas de observación, de manera que permitiera la explicación y valoración de resultados.

Dentro de los resultados encontrados sobresalían elementos comunes, tanto en problemáticas como en potencializadores del desarrollo, como por ejemplo: el desempleo, la migración de las personas jóvenes para estudiar y encontrar trabajo, la saturación y desvalorización de las actividades agropecuarias de la zona, la belleza escénica relacionada con sus montañas, cataratas, aves, sus campos sembrados y con animales, el volcán Turrialba, el Monumento Nacional Guayabo y ser la "cuna del queso tipo Turrialba".

El análisis de estos resultados permitió al equipo diseñar una propuesta que consideraba los elementos comunes como estrategia para potenciar el turismo rural comunitario.

Durante la aplicación de entrevistas y la observación se identificó a una organización que tenía dentro de sus objetivos fomentar el turismo en la zona, por lo que se invitó a las personas de la Unión de Grupos Organizados de Santa Cruz de Turrialba (Ugrosanta) a participar en la validación de los resultados presentados y la idea de una propuesta para contribuir en su transformación. Dicha organización se convertiría en la conexión del proyecto con actores locales durante los próximos tres años. 


\section{Construcción y ejecución de la propuesta}

El equipo de trabajo, a partir de la anuencia manifestada por parte de Ugrosanta de continuar el proceso de forma conjunta, se dio a la tarea de presentar la propuesta de elaboración de un Plan de Turismo Rural Comunitario, que ellos y ellas construyeran y ejecutaron, con el equipo de estudiantes, el SINAC y el cuerpo docente, como facilitador.

La metodología base es la investigación-acción participativa, ya que permite generar conocimiento colectivo que se nutre del conjunto de individualidades vinculadas. La diferencia principal de este tipo de investigación es la acción, entendida como el motor de la transformación, que en este caso consistió en el fortalecimiento de la organización, la promoción de la participación y la generación de capacidades para la autogestión de las y los integrantes de Ugrosanta.

Para Creswell, la investigación-acción participativa:

Posee tres fases esenciales: observar (que constituye la construcción de un diagrama del problema de estudio y la recolección de datos), pensar (comprende el análisis e interpretación de la construcción significante encontrada)y, la fase que caracteriza la investigación acción y que lo diferencia del resto de diseños cualitativos, el actuar (que engloba el momento en el cual la investigación busca la resolución de los problemas detectados mediante la implementación de mejoras). (Citado por Ramos, 2017, p. 13)

A continuación, las principales acciones realizadas durante los años de trabajo conjunto (Figura 1) y los resultados obtenidos (Figura 2):

Figura 1

Principales acciones realizadas

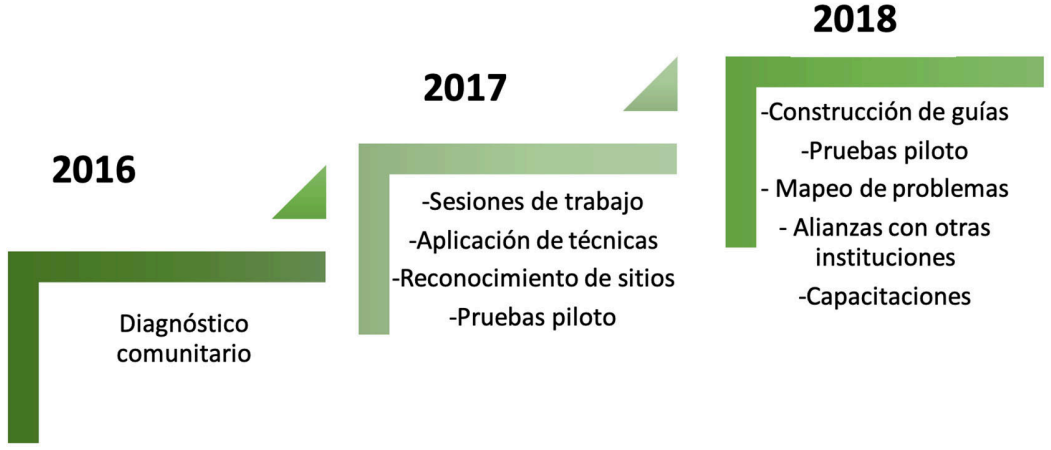

Nota. Elaboración propia 
URL: http://www.revistas.una.ac.cr/index.php/dialogo/index

CORREO ELECTRÓNICO: universidadendialogo@una.cr

DOI: http://doi.org/10.15359/udre.11-2.4

\section{Figura 2}

Principales resultados por año
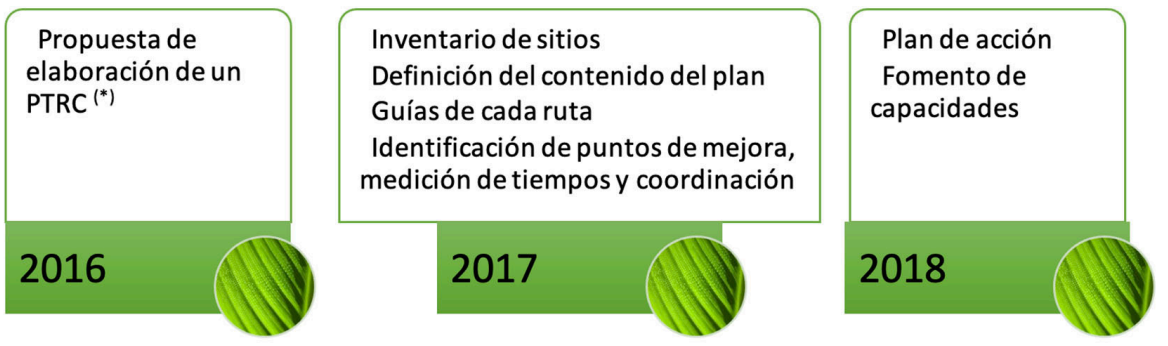

Nota. *Plan de Turismo Rural Comunitario. Elaboración propia

\section{Metodología utilizada en la elaboración del presente artículo}

Se desarrollan a continuación el paradigma, el enfoque, el tipo de investigación y las técnicas utilizadas para el desarrollo del presente artículo, según las características del proceso desarrollado.

El paradigma seleccionado fue el crítico social, al entenderse, según Valles (1999) y Sandoval (1997), citados por Carvajal (2005), como aquel que concibe la realidad epistémica considerando la cultura y las relaciones sociales, lo que permite, en esta investigación específica, un acercamiento y análisis de las formas de pensar y actuar de los actores sociales de la comunidad de Santa Cruz, del estudiantado, del profesorado y de las personas representantes de las instituciones y organizaciones.

Un aspecto clave es que una de las personas que figura como autora del presente artículo ha participado, durante los últimos cinco años, en iniciativas de la comunidad como facilitadora de procesos en pro del desarrollo de proyectos que las poblaciones consideran fundamentales, generando una relación sujetosujeto en donde se da un vínculo social entre la facilitadora, la comunidad, la población estudiantil y las instituciones, comprendiendo la subjetividad como medio de conocimiento.

Se buscó en esta investigación conocer la percepción de los diferentes actores participantes, por lo que el diseño de esta es flexible, entendiendo que la realidad es dinámica y compleja y que la validación de lo investigado se logra mediante el diálogo y consenso, en el que cada saber es clave para el análisis.

El enfoque bajo el cual se trabajó es el cualitativo, apoyado, además, en el estudio de caso que permitió examinar la interacción de factores en los procesos que 
Revista Universidad en Diálogo • Vol. 11, N. 2 2, Julio-Diciembre, 2021 • 69-94

ISSN 2215-2849 • EISSN: 2215-4752

URL: http://www.revistas.una.ac.cr/index.php/dialogo/index CorReo ElECTRÓNICO: universidadendialogo@una.cr DOI: http://doi.org/10.15359/udre.11-2.4

buscaban la transformación de las dinámicas organizacionales de Ugrosanta. El estudio se llevó a cabo apoyado en la percepción que tienen las personas de su propia realidad, lo que califican Bonilla y Rodríguez (1997), citados por Carvajal (2005), como captar la realidad "a través de los ojos de la gente que está siendo estudiada..." (p. 12). Ello facilitó incorporar las experiencias y los significados que dan los diferentes actores al aporte interinstitucional en los procesos de extensión que facilitan o no la transformación comunitaria.

Dadas sus características, la investigación cualitativa permitió generar hallazgos a partir de las reflexiones derivadas de un contexto general (la comunidad de Santa Cruz de Turrialba) y particular (integrantes de Ugrosanta, estudiantes, personas de las diferentes instituciones participantes), permitiendo aportar diferentes perspectivas y experiencias desde la interpretación y la percepción de los actores.

Particularmente, se recurre así a una investigación de tipo descriptiva que facilitó identificar las características del trabajo interinstitucional y sus aportes en procesos de desarrollo territorial.

Las fuentes de información en el desarrollo de esta producción académica fueron abordadas en tres niveles: desde lo institucional (universidades participantes, Sistema Nacional de Áreas de Conservación, Ministerio de Cultura, Juventud y Deportes), lo comunal y personas estudiantes que participaron activamente en el proceso de extensión.

Como técnicas de investigación se recurrió a la indagación documental, siendo los diagnósticos y las estrategias de desarrollo elaboradas por estudiantes de la Escuela de Planificación y Promoción Social en coordinación con los diferentes actores sociales la principal documentación indagada. Esta revisión permitió tener un acercamiento a aspectos tales como características de las comunidades, organizaciones de base e instituciones participantes en el proceso, durante el periodo 2016-2018.

Otra técnica utilizada fue la entrevista no estructurada, la cual se entiende como aquella que plantea "preguntas abiertas que son respondidas en una conversación teniendo como característica principal la ausencia de una estandarización formal" (Abarca et al., 2013, p. 107). Por lo que se entrevistó a personas de las instituciones involucradas en la experiencia, así como estudiantes y actores sociales pertenecientes a organizaciones comunitarias.

\section{Resultados}

Como resultado de las entrevistas realizadas a personas funcionarias de las instituciones que lideraron el proceso, se evidencia que reconocen como objetivo 
del proyecto el fortalecimiento comunal para el desarrollo y encadenamiento de iniciativas productivas o emprendimientos, particularmente asociados al turismo rural comunitario. Es así como objetivos claros y compartidos les permiten a las instituciones orientar de mejor manera los procesos y legitimar el trabajo con las comunidades.

Las instituciones también señalan como elementos a fortalecer: los conflictos generados a partir de la distribución o del acceso de recursos, la coordinación de intereses que estaban dispersos y los problemas de comunicación y atomización de estudios realizados por diversas instituciones sin resultados concretos ni devueltos a la comunidad.

El análisis de los resultados se evidencia en tres categorías: formas o prácticas de trabajo institucional, aportes del proceso de extensión universitaria y proceso de transformación comunitaria, familiar e individual; a su vez, cada categoría se analiza desde la mirada de las comunidades, de las personas funcionarias y del estudiantado participante en la experiencia.

\section{Formas o prácticas de trabajo interinstitucional}

\section{La mirada desde la comunidad}

- Las personas entrevistadas reconocen que las alianzas fueron importantes en el proceso, no solo se trabajó con la UNA, sino también con la Municipalidad, el Minae (así reconocen al Sinac), el Ministerio de Cultura y Juventud y el Tecnológico de Costa Rica (TEC).

- No se considera el trabajo de las instituciones de manera separada, sino que según la mirada de las personas todas iban por la misma línea de trabajo, un proceso integrado.

- Se esperaba que la participación de la Municipalidad fuera más activa, ya que fue solo en algunos momentos.

\section{Perspectiva desde las personas de instituciones participantes}

- Se reconoce, por parte de la UNA y del Sinac, la participación de diversas instituciones que dieron valor agregado y fortalecieron el alcance de los objetivos propuestos en el marco de las prácticas organizativas de la EPPS. Se mencionan: el Instituto Costarricense de Turismo (ICT), el Instituto Nacional de Aprendizaje (INA), el Colegio Universitario 
de Cartago (CUC), el Ministerio de Cultura y Juventud, las personas tesiarias del Centro Agronómico Tropical de Investigación y Enseñanza (Catie) y el Instituto de Desarrollo Rural (Inder).

- En menor medida, las instituciones participantes hacen referencia a una participación pasiva del gobierno local de Turrialba, a pesar de la gran importancia que reviste la Municipalidad en los ejercicios de desarrollo comunal.

- La alianza establecida entre el Sinac y la UNA, fortalecida por un trabajo continuo de tres años, se reconoce en las prácticas que se desarrollaron en torno al trabajo colaborativo: reuniones periódicas de coordinación y seguimiento, trabajo de campo llevado a cabo de manera conjunta, articulación de iniciativas de otras instituciones que aportaban en el proceso, inducción y acompañamiento de las personas estudiantes.

- Aun cuando cada institución brindó aportes puntuales de acuerdo con sus áreas de atención y su alcance, la labor conjunta y la articulación permitieron mantener el proceso por varios años.

- La existencia de iniciativas previas u organizaciones que lideran procesos en la comunidad facilita la inserción de otras iniciativas que, articuladas adecuadamente, potencian el recurso ya existente. El Ministerio de Cultura y Juventud había apoyado previamente el fortalecimiento organizacional de Ugrosanta, con la que desarrollaron una primera ruta turística.

- La madurez institucional para compartir información y experiencias es vital en procesos de articulación que permitan ir un paso más allá del trabajo y reconocimiento individual, y por el contrario potencian el trabajo colaborativo. La experiencia previa del Ministerio de Cultura y Juventud con Ugrosanta fortaleció el proceso desarrollado del 2016 al 2019.

- Si bien se espera la continuidad del proceso de un año a otro, la conformación del equipo de estudiantes varía y ello se percibió, por parte de las instituciones, como una afectación del avance de tareas puntuales, pues no siempre el grupo volvía a los resultados generados en años anteriores y ello implicó generar curvas de aprendizaje nuevas cada año. 


\section{Percepción del estudiantado que participó en la experiencia}

- La presencia institucional se reconoce, sin embargo, la articulación no se daba entre todas de la misma manera.

- La constancia en las intervenciones y los apoyos de las instituciones hacia la organización es necesaria para que el proyecto avance.

- El apoyo del Sinac a las personas estudiantes se reconoce como un elemento de incidencia en el éxito del proceso. Se trabajaba de manera conjunta metodologías y logísticas de trabajo de campo.

\section{Aportes del proceso de extensión}

\section{La mirada desde las comunidades}

- Se rescatan las capacitaciones como un aporte clave del proceso, entre las que se mencionan: trabajo en equipo, liderazgo, resolución de conflictos, definición del norte del proyecto, cómo identificar atractivos turísticos, estudios de costos, lo cual incluía cómo evaluar el producto que se iba a ofrecer a un precio justo para quien presta el servicio y para quien lo recibe también. Además, se realizaron visitas a otras iniciativas para compartir experiencias.

- Se elaboró el Plan de promoción, demanda y estrategia de mercadeo, con las posibles rutas de turismo.

- Existe una valorización de los aprendizajes, especialmente sobre turismo comunitario.

- La percepción sobre las personas funcionarias es excelente, sin embargo, se menciona que en el caso de una de las funcionarias les hablaba con un tono no adecuado y que los encuentros con las comunidades no deben ser así, porque esto ocasiona molestia en quienes participan.

- El papel del estudiantado es clave en estos procesos participativos, sin embargo, según personas entrevistadas, se evidenció que había quienes tenían poco entusiasmo y que consideraban las acciones de interés solo para cumplir con su curso de universidad. 


\section{Perspectiva desde las personas de instituciones participantes}

- Desde la institucionalidad se reconoce la interacción generada en varias vías: entre personas de la comunidad, entre la comunidad y las instituciones, entre instituciones. Incluso se reconoce con gran satisfacción la integración de personas estudiantes en familias de la comunidad, que permitió generar empatía y propiciar formas más accesibles para el trabajo conjunto.

- El fortalecimiento de capacidades surge como un elemento crítico mencionado ampliamente por las instituciones participantes: particularmente en competencias y habilidades blandas, como el trabajo en equipo.

- Sensibilización institucional y estudiantil respecto a las necesidades, pero también referente a las grandes oportunidades que presentan las comunidades y su aporte en el desarrollo territorial.

\section{Percepción del estudiantado que participó en la experiencia}

- Se mantuvo un esfuerzo constante por parte del sector estudiantil para atender los requerimientos de las personas en cuanto a información y asesoramiento.

- La articulación de metodologías de trabajo entre universidades y otras instituciones fue un ejercicio de negociación y construcción colectiva que benefició el proceso y representó mucho aprendizaje para las personas estudiantes.

- Se parte del conocimiento y de las necesidades de las personas de las organizaciones, por lo que el rol estudiantil consistió en acompañar y generar propuestas concretas que aprovechen sus capacidades y potencialidades como comunidad.

- Desde la extensión se apoyó el fortalecimiento de las estructuras organizativas de las agrupaciones a partir de las ideas e iniciativas que surgen desde las personas de la organización, del trabajo colectivo en pro del beneficio común. 
URL: http://www.revistas.una.ac.cr/index.php/dialogo/index

CORREO ELECTRÓNICO: universidadendialogo@una.cr

DOI: http://doi.org/10.15359/udre.11-2.4

\section{Proceso de transformación comunitaria, familiar e individual}

\section{La mirada desde las comunidades}

- Se considera el Plan de Turismo como un aporte al desarrollo comunal.

- Algunas personas mejoraron sus iniciativas, la cafetería, la soda, tanto en infraestructura como en servicio al cliente.

- Se señala que, aunque el proyecto no se continuó de manera grupal, las iniciativas se continuaron de manera individual, y que se notan los cambios, como en el caso del proyecto denominado: Taller de Tortillas.

- Algunas de las frases de las personas entrevistadas, según los aportes, son: "Este proceso nos ha dado confianza, me desenvuelvo mejor al atender a mis clientes", "aprendí muchísimo, me siento satisfecha", "logré la factura electrónica y aprender mucho sobre cómo manejar el negocio", "este proceso amplió mi visión sobre el turismo".

\section{Perspectiva desde las personas de instituciones participantes}

- Se identifica como cambio o transformación: mayor seguridad en la comunidad para el desarrollo de emprendimientos, apertura para alianzas estratégicas y encadenamientos, nuevos emprendimientos, así como una mayor identidad comunal.

- Desarrollo de un proceso de planificación participativo que respondiera a las necesidades expuestas por la comunidad.

\section{Percepción del estudiantado que participó en la experiencia}

- La puesta en práctica de los aprendizajes obtenidos en años anteriores de estudios, en contextos concretos, enriqueció la formación como planificadores con conciencia social y generó experiencias que permanecen en el tiempo.

- Este proceso permitió la reafirmación del compromiso con la carrera y el encuentro con la vocación, que se convierte en motivante durante 
los últimos años de carrera y será una experiencia importante en la vida profesional-laboral.

- Se propició el desarrollo de habilidades de comunicación, de negociación, de conciencia social a partir de la vinculación directa con comunidades, organizaciones e instituciones.

- Se reconoce a las personas de la comunidad de Santa Cruz como personas muy trabajadoras, con muchas ganas de mejorar sus condiciones actuales.

- Se genera un compromiso personal con los procesos comunales, gracias a los lazos que se construyen en los espacios de trabajo con las personas y al compartir con ellas y sus familias gracias a la hospitalidad y a la convivencia durante meses.

\section{Reflexiones}

1. Las iniciativas y los proyectos comunales deben pensarse como procesos dentro de un contexto articulado, que requiere de alianzas públicas y privadas.

2. El encadenamiento de las iniciativas de servicios y productos es clave para generar robustez y sostenibilidad.

3. El fin paralelo del trabajo institucional debe ser el fortalecimiento de las capacidades de autogestión de las organizaciones locales.

4. El gobierno local debe estar presente en los procesos que se estén impulsando desde el territorio, de manera que se incremente la articulación y viabilidad de las propuestas.

5. La estructura, la dinámica y el clima organizacional de las agrupaciones conformadas por las personas de la comunidad es determinante en el éxito del proceso.

6. Visualizar el bien común como elemento central de los procesos comunales acompañados por las instituciones debe ser el eje sobre el cual se realicen las decisiones y negociaciones.

7. El recurso económico es necesario, pero no indispensable para generar procesos exitosos en cuanto a ese bien común se refiere, si se cuenta con una agrupación comunal sólida, clara y comprometida. 
8. La incorporación activa del estudiantado contribuye con la generación de conciencia y compromiso social y crítico, por ello es clave que demuestren motivación e interés en los procesos, más allá de considerar esto como un curso o proceso de formación.

9. La formulación de planes de acción a partir de las lecciones aprendidas en los procesos de extensión y trabajo colaborativo promueve la calidad en los procesos de trabajo con las comunidades.

10.Trazar una estrategia que supere la visión de un año (espacio temporal de las prácticas organizativas) facilita la identificación de proyectos más sostenibles, contemplando una visión más integral y sistémica con participación de distintos actores.

11.Los plazos institucionales y los procesos administrativos no necesariamente coinciden con la realidad de las organizaciones y este es un problema importante que se visualiza desde la Administración Pública y que es necesario superar.

12.La continuidad de los procesos y proyectos comunitarios puede verse afectada por "celos profesionales" y paternalismo estatal. En la medida en que los actores participantes visualicen que la mejora en las condiciones de una de las partes repercute directamente sobre el todo, el territorio, la comunidad, la familia y lo individual, más efectivos y equilibrados serán los aportes, y más natural la articulación.

13. Si bien es cierto que los equipos de extensionistas integrados por el sector académico o estudiantil pueden dar recomendaciones a las personas de las comunidades, no deben olvidar que estos procesos participativos son encuentros de diálogo de saberes, no imposiciones que generen en las personas desmotivación.

14. 1 papel de la extensión universitaria debe ser la contribución a las rutas propias de transformación social planteadas por las comunidades, no desde los imaginarios de quienes llegan como actores externos.

15.Es fundamental construir un plan de trabajo de manera participativa y estar revisando los resultados con las personas de la comunidad, con el fin de ir marcando el avance o no del proceso y de esta manera determinar acciones. 


\section{Agradecimientos}

A las personas estudiantes que participaron a lo largo de todo el proceso, aportando entusiasmo y compromiso; a las personas de la agrupación Ugrosanta, que nos recibieron con cariño y confianza, de quienes guardamos recuerdos memorables de constancia y fuerza de trabajo en beneficio de la organización y de la comunidad; a las personas funcionarias, por el trabajo realizado y el esfuerzo de sobreponer el beneficio de las comunidades a los celos profesionales y las ansias de reconocimiento institucional. A todas estas personas que, además, amablemente atendieron la solicitud de ser entrevistadas para aportar a la elaboración de este artículo.

\begin{tabular}{lll}
\hline Estudantes: & Pessoas de Ugrosanta: & Funcionários públicos: \\
\hline Greivin Azofeifa Hernández & Miriam Corrales Castro & Gustavo Jiménez Blanco (Sinac) \\
Fabiola Barrantes Duarte & Rosa Fernández Coto & Mario Camacho Marín (MCJ) \\
Josué Meneses Reyes & Marjorie Mora Brenes & \\
& Rosa Torres Abarca & \\
& Cristina Casasola Pereira \\
\hline
\end{tabular}

\section{Referencias}

Abarca, A., Alpízar, F., Rojas, C. y Sibaja, G. (2013). Técnicas cualitativas de investigación. San José, Costa Rica: Editorial Universidad de Costa Rica.

Ávila, A. y Aguilar, A. (27-29 de mayo, 2020). Dinámica en la construcción de politicas de desarrollo territorial rural en la Región Chorotega, Costa Rica. [Ponencia]. V Congreso Internacional de Estudios del Desarrollo (V CIED). Desafíos al desarrollo: procesos de cambio hacia la justicia global. Bilbao.

Ávila, G. (2013). Desarrollo rural en Costa Rica: cuatro décadas después. Revista Perspectivas Rurales Nueva Época, 41-69. http://www.revistas. una.ac.cr/index.php/perspectivasrurales/article/view/5212/4970

Barragán de Anda, A. B., De Aguinaga, P. y Ávila, C. (2010). El trabajo colaborativo y la inclusión social. Apertura, 2(1). https://www.redalyc. org/pdf/688/68820841005.pdf

Benkler, Y. (2006). La riqueza de las redes, como la producción social transforma los mercados y la libertad. Icaria Editorial. https://web. archive.org/web/20150501025128/http://icariaeditorial.com/pdf libros $/ 1 \mathrm{a} \% 20$ riqueza\%20de\%20las\%20redes.pdf 
Bolívar, G. A. (2009). Sen y algunos escritos en la constitución del campo del Desarrollo Humano. Polis. http://journals.openedition.org/polis/1731

Carvajal, A. (2005). Elementos de investigación social aplicada. Escuela Latinoamericana de Cooperación y Desarrollo. Cartagena de Indias, Colombia.

Camacho, J. (2013). Desarrollo comunitario. Eunomía. Revista en Cultura de la Legalidad, N. ${ }^{\circ} 3,206-212$.

Carvajal, A. (Julio, 2011). Apuntes sobre desarrollo comunitario. Eumed.net, Universidad de Málaga-España, p. 83.

Dubois, A. (2014) Marco teórico y metodológico del Desarrollo Humano Local. Instituto de Estudios sobre el Desarrollo y Cooperación Internacional. Universidad del País Vasco, España.

Escuela de Planificación y Promoción Social. Facultad de Ciencias Sociales de la Universidad Nacional. (2019). Programa del curso Práctica Organizativa I.

Instituto de Desarrollo Rural. (2017). Plan Nacional de Desarrollo Rural Territorial 2017-2022.

Núñez, B. (1974). Hacia la universidad necesaria. Recuperado de http:// www. apeuna.una.ac.cr/index.php.

Organización de las Naciones Unidas (S.f). Objetivos de Desarrollo Sostenible. $\quad$ https://www.un.org/sustainabledevelopment/es/ objetivos-de-desarrollo-sostenible/

Programa de las Naciones Unidas para el Desarrollo. Desarrollo Humano. Informe 1990 (1990) Tercer Mundo Editores, Bogotá, Colombia. Recuperado de http://hdr.undp.org/sites/default/files/hdr 1990 es completo_nostats.pdf

Picazzo Palencia, E., Gutiérrez Garza, E., Infante Bonfiglio, J. M., \& Cantú Martínez,P.C.(2011).Lateoríadeldesarrollohumanoysustentable: hacia el reforzamiento de lasalud como un derechoylibertad universal. Estudios sociales (Hermosillo, Son.), 19(37), 253-279. http://www.scielo.org.mx/ scielo.php?script $=$ sci_arttext\&pid $=$ S0188-45572011000100010\&lng $=\mathrm{e}$ $\underline{\text { s\&tlng}=\mathrm{es}}$ 
Ramos, C. (2017). Los paradigmas de la investigación científica. Avances En Psicología, 23(1), 9-17. https://doi.org/10.33539/avpsicol.2015. $\underline{\mathrm{v} 23 \mathrm{n} 1.167}$

República de Costa Rica. (2017). Constitución Política de la República de Costa Rica [recurso electrónico] / comp. Vinicio Piedra Quesada. San José: Imprenta Nacional.

Ruiz, A. (2012). Diagnóstico de situaciones y problemas locales. Costa Rica: Editorial UNED.

Sánchez, M. (1989). Concepciones y políticas de extensión. Vicerrectoría de Extensión, Universidad Nacional.

Sepúlveda, S. (2008). Gestión del desarrollo sostenible en territorios rurales: métodos para la planificación. San José, Costa Rica: IICA.

Sepúlveda, S. y Zúñiga, H. (2008). Elementos conceptuales del desarrollo rural sostenible con enfoque territorial. En S. Sepúlveda, Gestión del desarrollo sostenible en territorios rurales: métodos para la planificación (pp. 1-103). San José, Costa Rica: IICA. http://repiica. iica.int/docs/B0712E/B0712E.pdf

Sobrado, M. (1976). Proyecto de Extensión Universitaria a Empresas Comunitarias Campesinas. [Ponencia] Seminario Latinoamericano "Formas Asociativas de Producción". 22-28 de noviembre de 1976. Bogotá, Colombia.

Universidad Nacional. (2016). Plan de Mediano Plazo Institucional 20172021. Rectoría, Comisión de Planificación. Universidad Nacional, Costa Rica.

Universidad Nacional. (2018). Dirección de Extensión UNA. www.extensión. una.ac.cr/index.php/. 